\title{
A study on comparison of retro-muscular pre-fascial placement of mesh versus other methods of mesh repair of ventral hernias
}

\author{
Darshan J. ${ }^{1}$, Nema A. ${ }^{2}$, Sheth J. ${ }^{3}$, Gohil J.A. ${ }^{4}$ \\ ${ }^{1}$ Dr Jitendra Darshan, Professor, ${ }^{2}$ Dr Archana Nema, Professor, ${ }^{3}$ Dr Jenish Sheth, Assistant Professor, ${ }^{4}$ Dr Jaypalsinh \\ Ashoksinh Gohil, Resident; all authors are affiliated with Department of Surgery, Surat Municipal Institute of Medical \\ Education and Research, Nr Bombay Market, Surat, Gujrat, India.
}

Corresponding Author: Dr Jitendra Darshan, Department of Surgery, Surat Municipal Institute of Medical Education and Research, Nr Bombay Market, Surat, India. E-mail-jr_darshan@yahoo.co.in

\begin{abstract}
Introduction: Ventral hernias repair are most routinely performed procedure in daily life of general surgeons. The objective of the present study is to compare the outcome of retro-muscular repair over other methods of ventral hernia repair. Methodology: 90 diagnosed cases of ventral hernias were randomly split into two groups A (retro-muscular meshplasty) and B (onlay, inlay \& underlay meshplasty). The comparison across groups was carried out in terms of operation length, postoperative pain, wound complications, length of hospital stay \& recurrence. Results: No difference was found between the groups regarding age, gender, type and classification of hernia. Operation length was 110 min in retro-muscular repair and 90min in onlay and 114 min underlay method. Statistically difference was seen between these groups. Among complications recurrence, seroma, mesh infection and wound complications were seen in group B. Postoperative pain and well being score were better in retro-muscular group. Conclusions: Retro-muscular meshplasty have more advantage compare to other open methods in ventral hernia repair. Retro-muscular meshplasty is still most appropriate method in open ventral hernia repair
\end{abstract}

Keywords: Ventral hernias, Meshplasty, Retro-muscular, Inlay, Onlay \& underlay meshplasty

\section{Introduction}

"No disease of the human body belonging to the province of the surgeon requires in its treatment a better combination of accurate anatomical knowledge with surgical skill than hernia in all its varieties" - Sir Astely copper (1804)

Abdominal wall hernias are a familiar surgical problem. Millions of patients are affected each year. Whether symptomatic or asymptomatic, hernias commonly cause pain or are aesthetically distressing to patients.

These concerns, coupled with the risk of incarceration, are the most common reason patient seek surgical repair of hernias.

More than 2 million laparotomies are performed annually with a reported 2 to $11 \%$ incidence of incisional hernia. It is most common complication after laparotomy by 2:1 ratio over bowel obstruction.

Manuscript Received: $22^{\text {th }}$ November 2018

Reviewed: $2^{\text {nd }}$ December 2018

Author Corrected: $8^{\text {th }}$ December 2018

Accepted for Publication: $14^{\text {th }}$ December 2018
Such hernias can occur after any type of abdominal wall incision although the highest incidence is seen with midline incision, the most common incision for many abdominal procedures [1-5].

The presence of ventral hernia is itself an indication for repair. The field of hernia repair has evolved as a result of surgical innovation and has benefited significantly from technologic improvement. The tension free repair is the key concept that has revolutionized hernia surgery.

The use of mesh prosthesis to approximate the fascial defect has resulted in a decrease in recurrence rates for inguinal and ventral hernias. More recently, Laparoscopic approaches to the inguinal and ventral hernias have extended the option and approaches for repairing the fascial defect. Modified Stoppa's repair includes placement of mesh in retro-rectus position with an excellent outcome in ventral hernia repair [6]. In the present study we aimed to compare the benefits of retrorectus meshplasty over other methods. 


\section{Original Research Article}

\section{Aims \& Objectives}

- To study the various presentations of ventral hernias.

- To standardize the preoperative preparation in patients of ventral hernias.
- To study the Intra-operative findings, type of mesh used, type of repair, drain requirements.

- To study the postoperative complications of meshplasty.

\section{Methodology}

Following approval of the institutional ethical committee, this longitudinal randomized single blinded comparative study was conducted in the Department of Surgery at SMIMER hospital, Surat during three and half years, from July'2011 till December'2014. A total of 90 patients of Ventral hernias were included in the study. 45 patients were randomly included in group A and rest in group B by using random number table method. Group A: included all patients operated by retrorectus meshplasty through midline vertical incision. Group B includes 45 patients operated by inlay, onlay, and underlay meshplasties.

Inclusion criteria: All patients above 18 years of age and both sex with diagnosed ventral hernia.

Exclusion criteria: Emergency condition like obstruction, incarceration, multiple lateral hernias, intra-operative complication like bowel injury was excluded.

Preoperatively all patients were assessed clinically and investigated thoroughly. All hernia repairs were deferred for at least one year from the last laparotomy.

Group A: All patients were operated with midline vertical incisions extending either side of umbilicus. The rectus sheath was opened at the edge of defect to dissect out the retro-rectus space. Hernia sac was opened in all cases and intraperitoneal contents assessed. Redundant sac was excised and defect, posterior rectus sheath along with peritoneum was closed in single layer with 1/0 polypropylene continuous sutures. An appropriate size of Polypropylene mesh was placed over posterior rectus sheath and fixed with $2 / 0$ propylene sutures. A mesh covered the defect and extended $5 \mathrm{~cm}$ beyond the margins of the defect or the umbilicus whichever was farthest from one end of defect. Rectus muscle was approximated without tension in midline. Anterior rectus sheath was approximated with continuous $1 / 0$ polypropylene sutures. Closed suction drain was placed in earlier few cases. Skin was the approximated.

Group B: Patients were operated by onlay 10 (22.2\%), and underlay 35 (77.8\%) meshplasty in standard conventional manner. In all overlay repairs the closed suction drain was used.

Post operatively all patients were monitored and recorded for vitals, bowel sounds, drain output. The post operative period for ambulation was recorded. Postoperative pain was recorded as per Visual Analogue scale which ranges from score 1 to 10 from 1 st postoperative day till $7^{\text {th }}$ day. Postoperative well being score was compared with WHO approved well being score from postoperative day 1 to 7 . Postoperative complications such as seroma/hematoma formation, sinus /fistula formation, flap necrosis were recorded and treated appropriately. Data was compared with "Chi Square Test" of significance between both groups.
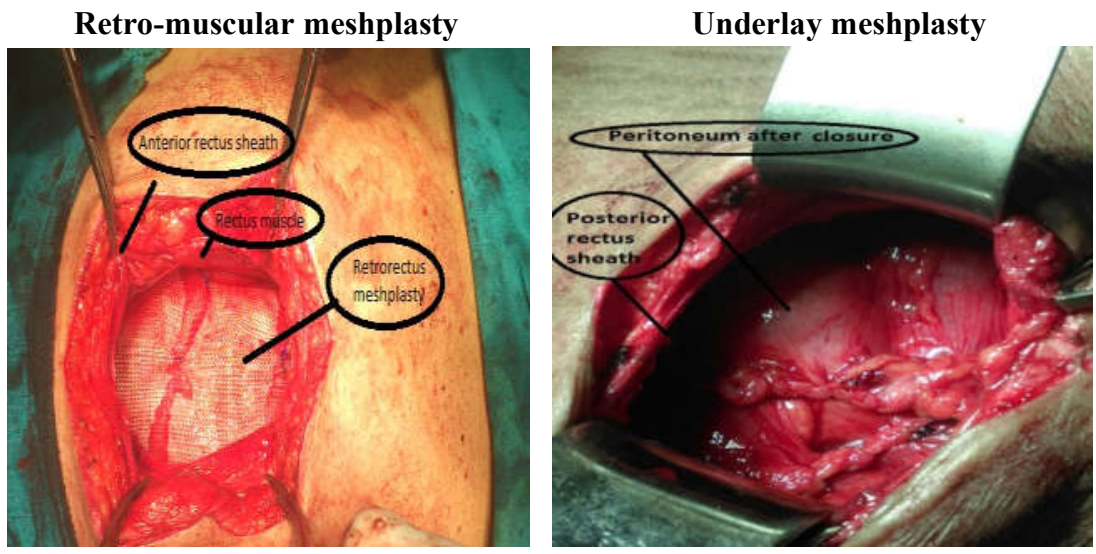

Figure-1: Retro-muscular space with mesh and pre-peritoneal (underlay) space 


\section{Original Research Article}

Patients were discharged from the hospital once drain was removed or when patient had no wound complications. Duration of post-operative hospital stay in number of days was recorded in both the groups and compared using "Leveny's T test "of significance. Patients were kept on regular follow ups for one year on telephone calls and personal interview and status of wound, any discomfort and recurrence were recorded.

\section{Results}

In this study of 90 patients, incisional hernia was mostly found in age group of 41 -50 years. Epigastric and Paraumbilical hernias were found in 21-40 years of age group. The majority of cases were of Post operative incisional hernia 22 (48.9\%) followed by Para-umbilical hernia 18 (40\%) in Group A whereas Para-umbilical hernia 19 (42.2\%) and Incisional hernia $17(37.8 \%)$ in group B. Majority of patient in each group were female, 23 in group A and 25 in group B. $\mathrm{P}-$ value $0.3(>0.05)$.

Hence there was no significant difference in distribution according to age and sex. The most common symptoms was abdominal swelling in both groups with 40 (88.9\%) in group A and 39 patient (86.7\%) in group B. Wound infection was the most common associated complication during previous surgery among the patients with post operative incisional hernia. It was seen in 5 patients (11.1\%) of group A and 14 patients $(20 \%)$ of group B. P-value $<0.05$ suggest significant association of previous wound infection with development of incisional hernia later.

Table-1: Duration of surgery

\begin{tabular}{|c|c|c|c|}
\hline \multirow{2}{*}{$\begin{array}{c}\text { Duration of Surgery } \\
\text { (MIN.) }\end{array}$} & $\mathbf{A}$ & \multirow{2}{*}{ Total } \\
\cline { 2 - 4 } & 0 & $1(2.2 \%)$ & $1(1.1 \%)$ \\
\hline 60 & 0 & $1(2.2 \%)$ & $1(1.1 \%)$ \\
\hline 75 & $26(57.8 \%)$ & $8(17.8 \%)$ & $34(37.8 \%)$ \\
\hline 90 & $10(22.2 \%)$ & $4(8.9 \%)$ & $14(15.6 \%)$ \\
\hline 110 & $7(15.6 \%)$ & $28(62.2 \%)$ & $35(38.9 \%)$ \\
\hline 120 & $2(4.4 \%)$ & $1(2.2 \%)$ & $3(3.3 \%)$ \\
\hline 150 & 0 & $2(4.4 \%)$ & $2(2.2 \%)$ \\
\hline 180 & $\mathbf{4 5}(\mathbf{1 0 0} \%)$ & $\mathbf{4 5 ( 1 0 0 \% )}$ & $\mathbf{9 0}(\mathbf{1 0 0} \%)$ \\
\hline
\end{tabular}

P-value- $<0.0001$

Patients operated by retro-muscular meshplasty have duration of surgery less (M-110min) compare to underlay meshplasty (M-114 min) but higher compared to onlay (M-90 min.) meshplasty method. P-value $-<0.0001$ (M-mean).

Table-2: Complication

\begin{tabular}{|c|l|c|c|c|}
\hline S No & Complication & $\mathbf{A}(\mathbf{n}=\mathbf{4 5})$ & $\mathbf{B}(\mathbf{n}=\mathbf{4 5})$ & P-value \\
\hline 1 & Drain used & $23(51.11 \%)$ & $45(100 \%)$ & $<0.0001$ \\
\hline 2 & Seroma & 0 & $08(17.78 \%)$ & $0.006(<0.05)$ \\
\hline 3 & Hematoma & 0 & $06(13.33 \%)$ & $0.026(<0.05)$ \\
\hline 4 & Deep (Mesh) Infection & 0 & $01(2.22 \%)$ & $0.5(>0.05)$ \\
\hline 5 & Wound Infection & 0 & $02(4.44 \%)$ & $0.2(>0.05)$ \\
\hline 6 & Recurrence & 0 & $2(4.44 \%)$ & $0.24(>0.05)$ \\
\hline
\end{tabular}

Drain was used in all 45 patients of group B while 23 patients in group A required negative suction drain. (P-value $<0.0001)$. 08 cases developed seroma in group B whereas none in group A. Hematoma was seen in 6 patients $(13.30 \%)$ in group B in and none in group A. P-value $0.026(<0.05)$ which is significant. Mesh infection was seen in 01 patient in group $\mathrm{B}$, in underlay mesh repair. There was no case of mesh infection in patient operated by retro-muscular meshplasty and onlay meshplasty. Wound infection was seen in group B patients only, among these 2 patients were operated with onlay meshplasty and 1 patient with underlay meshplasty. P-value $0.2(>0.05)$. There was no recurrence in group A and in group B there were 2 recurrences, 1each in patients operated by onlay and underlay meshplasties respectively. P-value $-0.24(>0.05)$. 


\section{Original Research Article}

Table-3: Comparison of post operative pain (Mean value of VAS scale)

\begin{tabular}{|l|c|c|c|c|c|c|c|c|}
\hline Post Op Day & \multicolumn{2}{|c|}{1} & \multicolumn{2}{c|}{2} & \multicolumn{2}{|c|}{3} & \multicolumn{2}{c|}{4} \\
\hline Group & A & B & A & B & A & B & A & B \\
\hline Mean VAS & 3.13 & 5.18 & 1.96 & 3.89 & 0.80 & 2.87 & 0.09 & 1.89 \\
\hline SD & 0.919 & 1.336 & 0.737 & 0.959 & 0.625 & 0.757 & 0.288 & 0.682 \\
\hline p- value & \multicolumn{2}{|c|}{$\mathrm{P}<.0001$} & \multicolumn{2}{|c|}{$\mathrm{P}<0.0001$} & \multicolumn{2}{c|}{$\mathrm{P}<0.0001$} & \multicolumn{2}{c|}{$\mathrm{P}<0.0001$} \\
\hline
\end{tabular}

VAS value revealed Retro-muscular meshplasty was significantly less painful compared to onlay and underlay meshplasty. P-value $<0.0001$. After 5 days there was no significant difference between two groups.

Table-4: Mean value of well being score

\begin{tabular}{|l|c|c|c|c|c|c|c|c|c|c|}
\hline \multirow{2}{*}{$\begin{array}{l}\text { Well } \\
\text { being } \\
\text { Score }\end{array}$} & \multicolumn{2}{|c|}{1} & \multicolumn{2}{c|}{2} & \multicolumn{2}{c|}{3} & \multicolumn{2}{c|}{4} & \multicolumn{2}{c|}{5} \\
\cline { 2 - 11 } & A & B & A & B & A & B & A & B & A & B \\
\hline Mean & 9.578 & 6.556 & 12.444 & 9.0222 & 15.311 & 11.644 & 20.422 & 15.067 & 21.489 & 19.356 \\
\hline SD & 2.9885 & 3.4939 & 3.0567 & 3.4476 & 2.6784 & 3.6937 & 1.2521 & 3.4470 & 0.5055 & 3.7849 \\
\hline p- value & \multicolumn{2}{|c|}{$\mathrm{P}<.0001$} & \multicolumn{2}{|c|}{$\mathrm{P}<0.0001$} & \multicolumn{2}{c|}{$\mathrm{P}<0.0001$} & $\mathrm{P}<0.0001$ & \multicolumn{2}{c|}{$\mathrm{P}<0.001$} \\
\hline
\end{tabular}

Mean well being score was in higher on each post operative day in group A compared to group B from first to fifth post operative day, P-value $<0.0001$ suggesting significant difference and patient operated by retro-muscular meshplasty have rapid recovery. After 6th post operative day well being score was within equal range in both groups

\section{Discussion}

Ventral hernia operations are still one of the most commonly encountered procedures in the lifetime of a general surgeon. In ventral hernia surgery, the best indicator of the success of the operation is the recurrence which totally based on objective criteria. In this study of 90 cases, patients were followed up-to 1 year. Recurrence was categorised as early ( $<1$ year) and late. Tension in the reinforced line is held responsible for the early recurrence, disruption in the collagen metabolism is held accountable for late recurrences.

The use of synthetic non absorbable sutures for abdominal wall closure provides significant tensile strength and delays development of swelling from date of reparative surgery. In most circumstances this strength is sufficient to hold the fascia together, but with delayed healing due to infection or raised intraabdominal pressure, postoperative chest infection or abdominal distension the strength of the wound may be insufficient, leading to the formation of an incisional hernia [7]. This is comparable with Obey et al [8], where $20-30 \%$ wounds were infected. Approximately $35 \%$ to $40 \%$ of incisional hernias occur with a documented history of wound infection. Another study reported incidence of wound infection varying from 5 to $20 \%$ [9]. In this study total $14(15.6 \%)$ patient had history of wound infection during previous surgery. As per Toms P.A. et al [10], incisional hernias are more common following midline incision through relatively avascular linea alba.
Previous wound complication: Blomstedt and Bauer 1972 et al [11] demonstrated that Post operative wound infection was associated with a fivefold increase in the risk of development of hernia (23\%) compared with patients with uninfected wounds.

Duration of surgery: The length of operative time should be kept to minimum which is important in respect to hernia surgery. Reduced operative time especially in patients who are middle aged to elderly reduces the risk of associated complication. The mean time to perform retro-muscular meshplasty in this study group A was 110 minutes and group B was 146 minutes with $\mathrm{P}$ value $<0.0001$, suggestive of significant difference in duration of surgery between group A and Group B. The difference of time can be accounted due to more dissection needed for creating preperitoneal space. Onlay meshplasty has mean duration of surgery of 90 minutes, because it requires less tissue dissection. Duration of surgery is further affected by large hernias and multiple adhesions where it takes more time for tissue dissection. Patient operated by underlay meshplasty had mean operative time of 120 minutes. 3 patients in group B operated by underlay meshplasty had duration of surgery up to 150 minutes. These results are comparable with other studies FS Aoda [12] and Polemon et al [13] both of which showed mean duration of surgery for onlay meshplasty was 63 minutes. In retro-muscular meshplasty it takes time for dissection of hernial sac and identification of defect margins. 


\section{Original Research Article}

Once defect margins are identified it is easier to separate posterior rectus sheath from anterior rectus sheath \& rectus muscle and making retro-muscular space. This is also supported by V Opera et al [14] where mean duration of surgery was 110 minutes.

Wound complications: Group A patients had no incidence of post hernia repair wound infection, seroma or hematoma formation, but in group B three patients had wound infection. Among this 1 patient had intraabdominal abscess, operated by underlay meshplasty. 2 patients had wound infection operated by onlay meshplasty. P-value was $>0.05$ which is not significant and indicates wound infection can occur in both groups if proper aseptic precautions were not taken. Complications like seroma formation, hematoma, wound infection attributed largely to extensive dissection and tissue handling during hernia repair.

Patients operated by onlay meshplasty had higher incidence of wound complication because it requires flap dissection in subcutaneous tissue plain with extensive dissection. This is comparable with study of Luijendijk et al [15] having seromas formation in 4 cases. Wound infection was seen in 3 patient in Luijendijk et al. and 3 patients in Korenkov M [16].

Post operative pain \& well being score: In this study, pain was scored according to VISUAL ANALOGUE SCALE [17] from post operative day 1 to 7 . According to Whitney $\mathrm{T}$ test, $\mathrm{P}$ value was $<0.05$ from post operative day 1 to 4 . This indicates patients operated by retro-muscular meshplasty having less post operativepain level compared to group B. Less post operative pain may be due to less tissue dissection and proper tissue handling. In this study, well being score was measured according to WHO APPROVED WELL BEING SCORE BY ASSOCIATION OF PSYCHIATRIST [18] from post operative 1 to 7 days, ranging from $0-25$.

According to $\mathrm{t}$ test, $\mathrm{p}$ value was $<0.05$ from post operative day 1 to 5 , which indicates that patient operated by retro-muscular meshplasty had higher mean well being score compared to patient operated by onlay/ underlay meshplasty. Well being score was good in group A because of less post operative pain and no drain placement and if drain kept than early removal, compared to group B.

Use of mesh: When choosing a mesh the surgeon must consider the context in which it is to be used. In most situations, one should look for a light weight mesh, with large pores and minimal surface area. Ideally it should consist of a monofilament non irritant material. Klinge et al [19] found that light weighted polypropylene mesh exhibit better tissue integration. Heavy weighted mesh induces greater tissue inflammatory response, scar formation, wound contracture and greater pain. Polypropylene mesh is ideal for meshplasty. In this study polypropylene mesh was used in all cases.

Drain and its removal: In this study, in group B, all cases required negative suction drainage because of more tissue dissection. In group $\mathrm{A}$, not all but some initial cases required negative suction drain, because mesh kept in retro-muscular space, where muscles having good absorptive surface. Mean duration of drain removal was 2-3 days in group A while 4-5 days in group B.

F S Aoda et al [12] recorded negative suction drain kept in all cases. Duration of removal of drain was $2^{\text {nd }}$ to $5^{\text {th }}$ postoperative day in patient operated by onlay meshplasty, and was $3^{\text {rd }}$ to $5^{\text {th }}$ postoperative day in patients operated by underlay meshplasty.

Duration of hospital stay: Mean duration of post operative hospital stay in this study was 5.22 days (SD 0.6) for group A and 7.42 days (SD 0.9) for group B with $\mathrm{P}$-value $<0.0001$, which is significant. It indicates there was a significant difference in mean hospital stay in both groups. Patient operated by retro-muscular meshplasty had shorter postoperative hospital stay compared to patient operated by onlay/ underlay meshplasty, because longer duration of drain requirement. This is also supported by FS Aoda et al ${ }^{[12]}$ where average duration of hospital stay was 8 days for patients operated by onlay meshplasty, and 7 days for patients operated by underlay meshplasty.

\section{Conclusion}

Meshplasty is the treatment of choice for ventral hernia repair. Polypropylene mesh was used in all cases. Post operative wound infection is important cause for development of incisional hernias. Results of the study showed that implantation of polypropylene mesh using the retro-muscular technique is safe and effective in the treatment of ventral abdominal hernias.

This technique allows patients to recover fast with low level of postoperative pain, less post operative complications and low recurrence rate, compared to onlay / underlay meshplasty. Mean duration of surgery is less in retro-rectus meshplasty compared to onlay/underlay meshplasty. Duration of hospital stay 


\section{Original Research Article}

also less (5 days) in patient operated by retro-rectus meshplasty compared to onlay/underlay meshplasty. Patient operated by retro-rectus meshplasty having less postoperative pain. Patient operated by retro-rectus meshplasty have overall better well being score compared to patients operated by onlay / underlay meshplasty.

Conflict of interest: None declared.

Funding: Nil, Permission from IRB: Yes

\section{References}

1. Bucknall TE, Cox PJ, Ellis H. Burst abdomen and incisional hernia: a prospective study of 1129 major laparotomies. Br Med J (Clin Res Ed). 1982 Mar 27; 284 (6320): 931-3.

2. George CD, Ellis H. The results of incisional hernia repair: a twelve year review. Ann R Coll Surg Engl. 1986 Jul;68(4):185-7.

3. Luijendijk RW ${ }^{1}$, Hop WC, van den Tol MP, et al. A comparison of suture repair with mesh repair for incisional hernia. N Engl J Med. 2000 Aug 10;343(6): 392-8. DOI:10.1056/NEJM200008103430603

4. Burger JW, Luijendijk RW, Hop WC, et al. Longterm follow-up of a randomized controlled trial of suture versus mesh repair of incisional hernia. Ann Surg. 2004 Oct; 240(4):578-83; discussion 583-5.

5. Mudge M, Hughes LE. Incisional hernia: a 10 year prospective study of incidence and attitudes. $\mathrm{Br} \mathrm{J}$ Surg. 1985 Jan;72(1):70-1.

6. Anthony $\mathrm{T}$, Bergen PC, Kim LT, et al. Factors affecting recurrence following incisional herniorrhaphy. World J Surg. 2000 Jan;24(1):95-100; discussion 101.

7. Bucknall TE, Cox PJ, Ellis H. Burst abdomen and incisional hernia: a prospective study of 1129 major laparotomies. Br Med J (Clin Res Ed). 1982 Mar 27; 284(6320):931-3.

8. OBNEY N. An analysis of 192 consecutive cases of incisional hernia. Can Med Assoc J. 1957 Sep 1;77(5): 463-9.
9. Condon RE. Ventral Abdominal Hernia 2001. In Baker RJ, Fischer JE editors Mastery of Surgery 4th edition. Philadelphia: Lippincott Williams \& Wilkins.

10. Toms AP, Dixon AK, Murphy JM, et al. Illustrated review of new imaging techniques in the diagnosis of abdominal wall hernias. Br J Surg. 1999 Oct;86 (10) : 1243-9.

11. Bauer JJ, Salky BA, Gelernt IM, et al. Repair of large abdominal wall defects with expanded polytetrafluoroethylene (PTFE). Ann Surg. 1987 Dec; 206 (6):765-9.

12. FS Aoda et al. Underlay versus onlay mesh repair of ventral hernias. QMJ VOL.9 No.16, p-208-216.

13. Poelman MM, Langenhorst BL, Schellekens JF, et al. Modified onlay technique for the repair of the more complicated incisional hernias: single-centre evaluation of a large cohort. Hernia. 2010 Aug;14(4):369-74. doi: 10.1007/s10029-010-0646-3. Epub 2010 Mar 14.

14. Cassar K, Munro A. Surgical treatment of incisional hernia. Br J Surg. 2002 May;89(5):534-45.

15. Luijendijk RW, de Lange DC, Wauters CC, et al. Foreign material in postoperative adhesions. Ann Surg. 1996 Mar;223(3):242-8.

16.Korenkov M, Beckers A, Koebke J, et al. Biomechanical and morphological types of the linea alba and its possible role in the pathogenesis of midline incisional hernia. Eur J Surg. 2001 Dec;167(12):909-14. DOI10. 1080/110241501753361596

17. Peter Tyrer. Classification of pain \& Description of pain. Cambridge textbook of effective treatments in psychiatry. Vol 3, p151-185.

18. Peter Tyrer. Classification of well being score \& Description. Cambridge textbook of effective treatments in psychiatry. Vol 3, p351-385.

19. Welty $\mathrm{G}^{1}$, Klinge $\mathrm{U}$, Klosterhalfen $\mathrm{B}$, et al. Functional impairment and complaints following incisional hernia repair with different polypropylene meshes. Hernia. 2001 Sep;5(3):142-7.

\section{How to cite this article?}

Darshan J., Nema A., Sheth J., Gohil J.A. A study on comparison of retro-muscular pre-fascial placement of mesh versus other methods of mesh repair of ventral hernias. Surgical Update: Int J surg Orthopedics.2018;4(4):177182.doi:10.17511/ijoso.2018.i04.08. 